\title{
Imaging Postoperative Abdominal Hernias: A Review with a Clinical Perspective
}

\author{
Argha Chatterjee $^{1}$ Rochita V. Ramanan² Sumit Mukhopadhyay ${ }^{2}$
}

\author{
${ }^{1}$ Department of Radiology and Imaging, Tata Medical Center, \\ Kolkata, West Bengal, India \\ ${ }^{2}$ Department of Radiology, Apollo Hospital Chennai, Chennai, India

\begin{abstract}
Address for correspondence Argha Chatterjee, MD, Department of Radiology and Imaging, Tata Medical Center, Kolkata, West Bengal, India (e-mail: arghachat84@gmail.com).
\end{abstract}

\begin{abstract}
Postoperative internal hernia is a challenging but critical diagnosis in postoperative patients presenting with acute abdomen. Postoperative internal hernias are increasingly being recognized after Roux-en-Y gastric bypass (RYGB) and bariatric surgeries. These internal hernias have a high risk of closed-loop obstruction and bowel ischemia; therefore, prompt recognition is necessary. Computed tomography (CT) is the imaging modality of choice in cases of postoperative acute abdomen. Understanding the types of postoperative internal hernia and their common imaging features on $\mathrm{CT}$ is crucial for the abdominal radiologist. Postoperative external hernias are usually a result of defect or weakness of the abdominal wall created because of the surgery. CT helps in the detection, delineation, diagnosis of complications, and surgical planning of an external hernia. In this article, the anatomy, pathophysiology, and CT features of com-

Keywords

- internal hernia

- incisional hernia

- computed tomography mon postoperative hernias are discussed. Afterreading this review, the readers should be able to (1) enumerate the common postoperative internal and external abdominal hernias, (2) explain the pathophysiology and surgical anatomy of Roux-en-Y gastric bypass-related hernia, (3) identify the common imaging features of postoperative hernia, and (4) diagnose the complications of postoperative hernias.
\end{abstract}

\section{Introduction}

Postoperative internal hernia remains one of the most challenging but critical diagnoses in postoperative patients presenting with an acute abdomen. Postoperative internal hernias are increasingly being encountered especially with the increase in the number of Roux-en-Y gastric bypass (RYGB) and bariatric surgeries. ${ }^{1}$ In postoperative bariatric patients, internal hernias are one of the most common postoperative complications, reported in 2 to $4.5 \%$ of patients. ${ }^{2,3}$ Internal hernias following RYGB surgery have a high risk of closed-loop obstruction and bowel ischemia, and consequently, they require prompt recognition and surgical treatment. ${ }^{4}$ The radiological diagnosis of any internal hernia remains difficult. Although fluoroscopy with a water-soluble contrast agent is routinely employed in the immediate postoperative period to rule out an anastomotic leak, computed tomography (CT) is the imaging modality of choice in cases of postoperative acute abdomen..$^{4-6} \mathrm{~A}$ high index of clinical suspicion in postoperative patients is necessary for a prompt and accurate diagnosis. Understanding the types of postoperative internal hernia and their common imaging features on $\mathrm{CT}$ is crucial for the abdominal imagers.

Postoperative external hernias are usually a result of defect or weakness of the abdominal wall created because of the surgery. These hernias are often clinically evident. However, incidental detection of an incisional hernia in an asymptomatic patient is common in routine postoperative CT. CT helps in the detection and estimation of size, site, number, and complications of an external hernia. Abdominal CT helps in surgical planning before incisional hernia repair.
DOI https://doi.org/ $10.1055 / \mathrm{s}-0040-1715772$ ISSN 2581-9933.
C 2020. Indian Society of Gastrointestinal and Abdominal Radiology. This is an open access article published by Thieme under the terms of the Creative Commons Attribution-NonDerivative-NonCommercial-License, permitting copying and reproduction so long as the original work is given appropriate credit. Contents may not be used for commercial purposes, or adapted, remixed, transformed or built upon. (https://creativecommons.org/licenses/by-nc-nd/4.0/) Thieme Medical and Scientific Publishers Pvt. Ltd., A-12, 2nd Floor, Sector 2, Noida-201301 UP, India 


\section{Classification}

- Internal hernias

- RYGB associated hernia

- Retro-colic/retroperitoneal hernia

- Perineal hernia

- External hernias

- Incisional herma

- Parastomal hernia

- Port-site associated hernia

\section{Internal Hernia}

Postoperative internal hernias result from the protrusion of small bowel through an abnormal aperture created by surgery. The aperture can be of three types: first, a mesenteric defect that results from the anastomosis of two segments of the gastrointestinal tract, especially the small bowel; second, adhesive peritoneal bands that are created because of postoperative inflammation and subsequent healing; and finally, an abnormal opening in mesentery created deliberately during surgery, such as the hole created in transverse mesocolon in retrocolic RYGB surgery (-Fig. 1). Some authors also consider postoperative band related obstruction as a fourth type of internal hernia; however, since there is no true aperture, we have not considered this type of small-bowel obstruction as a true internal hernia.

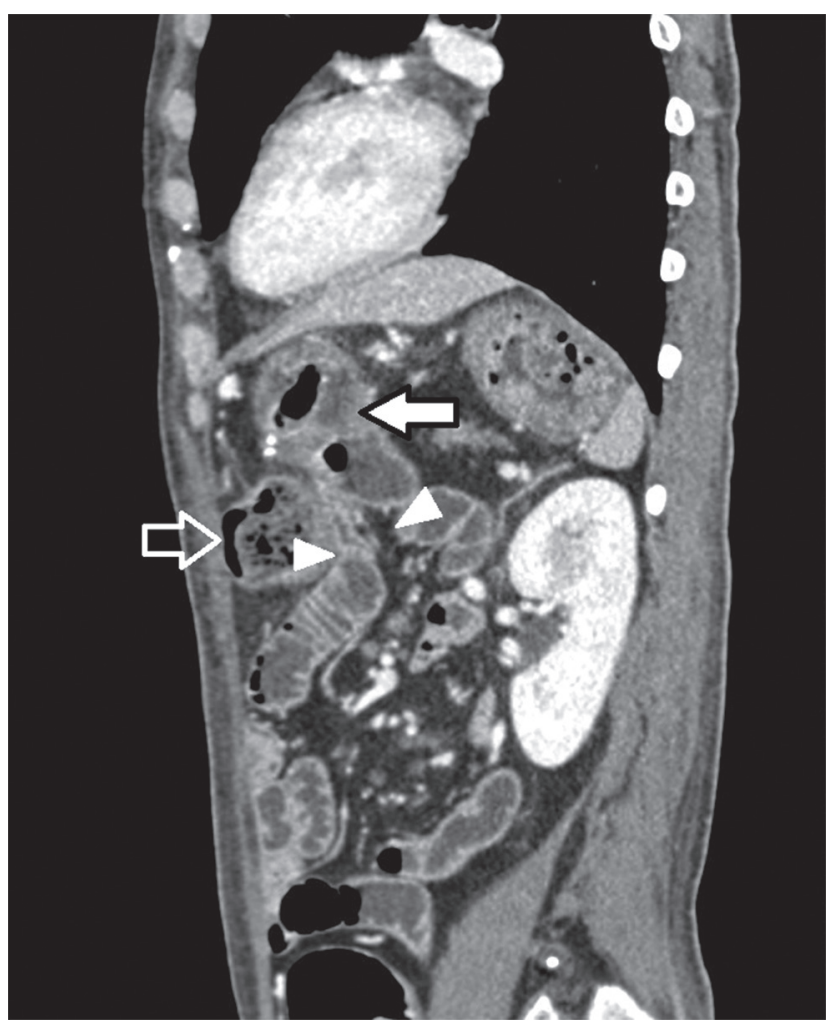

Fig. 1 Postoperative anatomy. Sagittal reformation of contrastenhanced computed tomography shows the transverse colon in cross-section anteriorly (open arrow) with the jejunal loop that is pulled through a defect (between arrowheads) in the transverse mesocolon to create an anastomosis with the posterior wall of stomach (closed arrow). Therefore, this is a retrocolic posterior gastrojejunostomy. Knowledge of the postoperative defects is important to understand the pathological anatomy of postoperative internal hernia.

\section{General Considerations}

Internal hernia is prone to twisting of the mesentery and a volvulus formation that gives rise to the "Swirl sign" on CT. This is found to be the most sensitive sign of internal hernia following gastric bypass ( - Fig. 2). ${ }^{6}$ However, this sign is not specific and is found in mesenteric volvulus or twist from any cause. The most specific feature of an internal hernia is the presence of sac-like clustering of the small bowel. ${ }^{7}$ The saclike clustering is seen when the bowel loops pass between the two leaves of the mesentery (intramesenteric hernia; -Fig. 3). A closed-loop is identified by a beak-like narrowing at two ends of the bowel loop ( - Fig. 4 ) and the radial arrangement of the mesenteric vessels. ${ }^{8}$ The flaring of the bowel loops immediately after the beak-like narrowing is sometimes called the "mushroom sign." This finding is best seen in multiplanar reconstruction of CT along the plane parallel to the mesentery of the affected bowel loops. ${ }^{7}$ General features that should raise the suspicion of postoperative internal hernia are given in - Table $\mathbf{1}$. The hernial orifice can be identified by identifying the point where the mesenteric vessels of the closed-loop converge ${ }^{9}$ (-Fig. 5).

\section{Roux-en-Y Gastric Bypass Associated Hernia}

RYGB surgeries are one of the most common bariatric surgeries where stomach is converted to a small pouch that is connected to the jejunum directly. Other than as a weight loss procedure, gastric bypass is also performed in cases of benign and neoplastic gastric outlet obstruction.

In RYGB, a small gastric pouch is created with the residual stomach. The jejunum is divided 25 to $50 \mathrm{~cm}$ distal to the ligament of Treitz, and the distal component is brought up and anastomosed to the gastric remnant (the G-J anastomosis). The distal component is called the Roux limb or the efferent limb. The Roux limb may be brought up anterior to (antecolic) or through (retrocolic) the transverse mesocolon. Finally, the afferent limb is anastomosed with the roux loop 75 to $150 \mathrm{~cm}$ distal to the G-J anastomosis in side-to-side

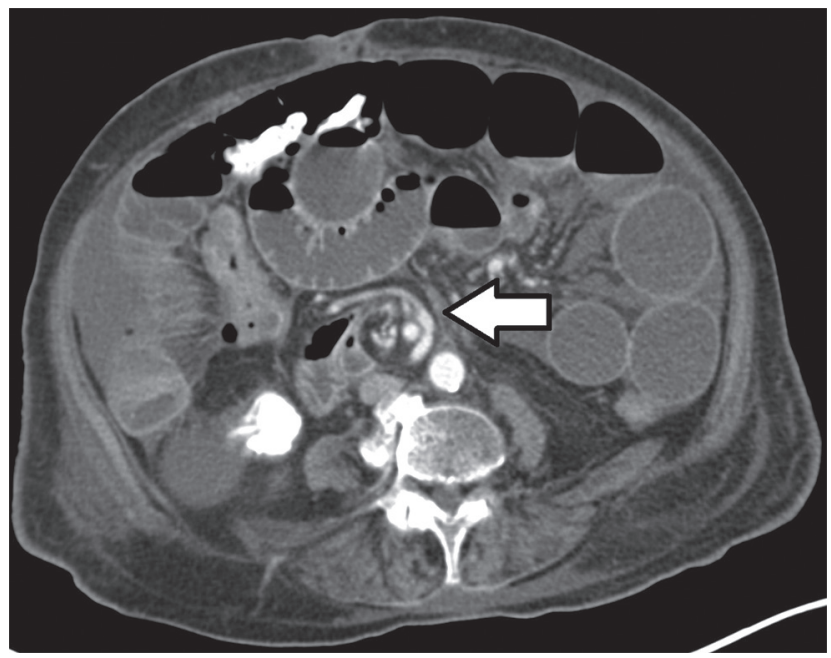

Fig. 2 Swirling mesentery. Axial contrast-enhanced computed tomography shows swirled appearance of the mesenteric vessels in the right lower quadrant around the superior mesenteric arterial pedicle; the so-called "swirl sign" (arrow). 


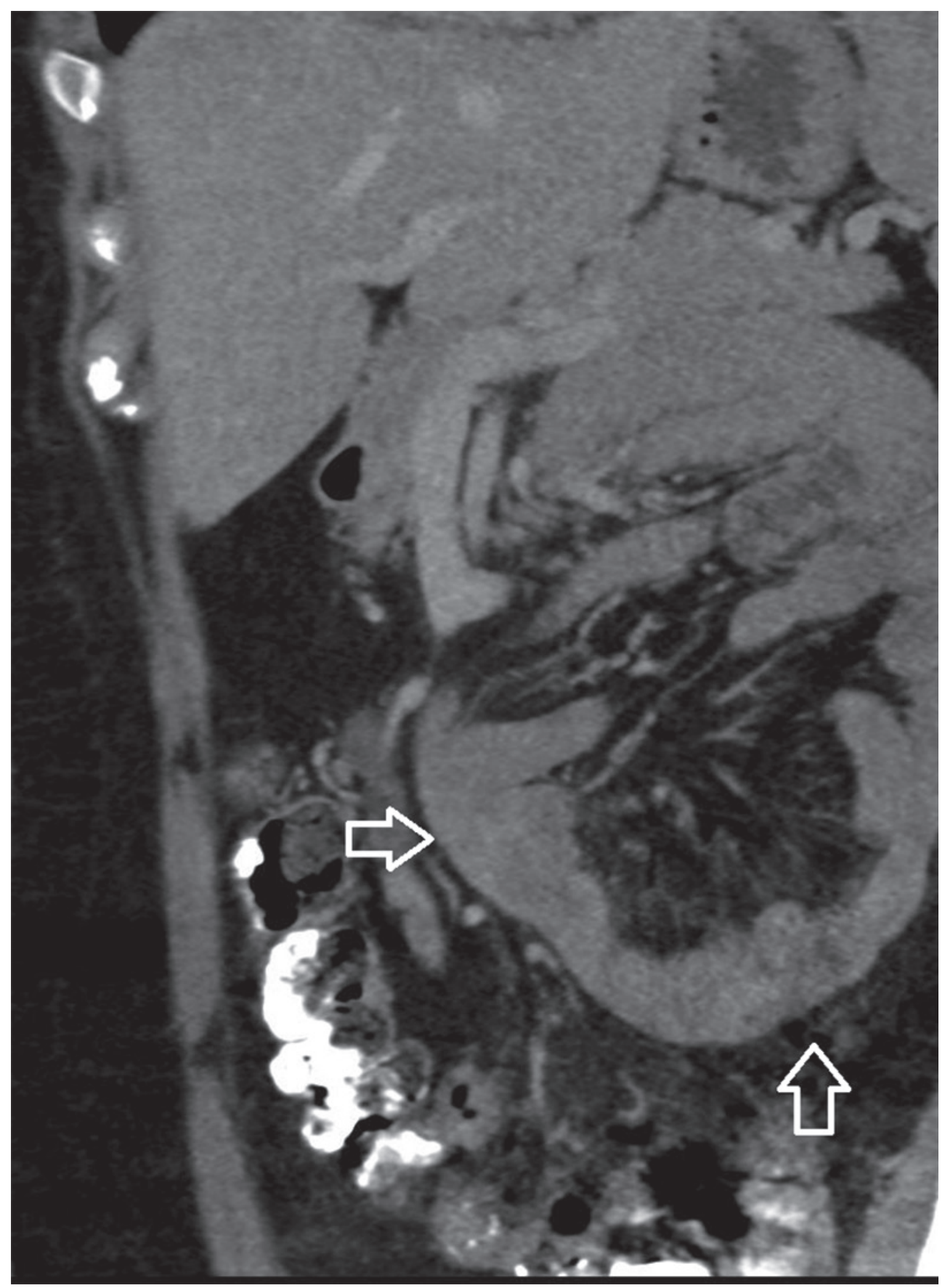

Fig. 3 Sac-like appearance of internal hernia. Oblique reformation of contrast-enhanced computed tomography shows a large internal hernia containing small bowel loops. Notice the outer walls of the small bowel loops conform to form a smooth "sac-like" appearance (arrows).

fashion (the entero-enterostomy or J-J anastomosis). RYGB can be performed laparoscopically or as an open surgical procedure. The incidence of internal hernia is more after the laparoscopic approach. This is probably because the laparoscopic approach results in fewer adhesions leading to unrestricted mobility of the bowel loops enabling internal herniation ${ }^{2}$. Even though the most common cause of small bowel obstruction after open RYGB is postoperative adhesions, the most common cause of the same after laparoscopic RYGB is internal hernia. ${ }^{10}$ While RYGB remains a relatively common bariatric procedure in the United States, other bariatric surgical procedures like laparoscopic sleeve gastrectomy have become increasingly popular in recent years due to their comparable efficacy and the lower reported incidence of complications, including internal hernias. ${ }^{11}$

\section{Imaging Technique}

$\mathrm{CT}$ is the mainstay for the diagnosis of postoperative internal hernia. ${ }^{5}$ A small volume $(200-250 \mathrm{~mL})$ of oral contrast material immediately before the $\mathrm{CT}$ can facilitate the identification of the Roux limb and delineation of the surgical anatomy. ${ }^{12,13}$ However, patients presenting with acute abdomen or features 


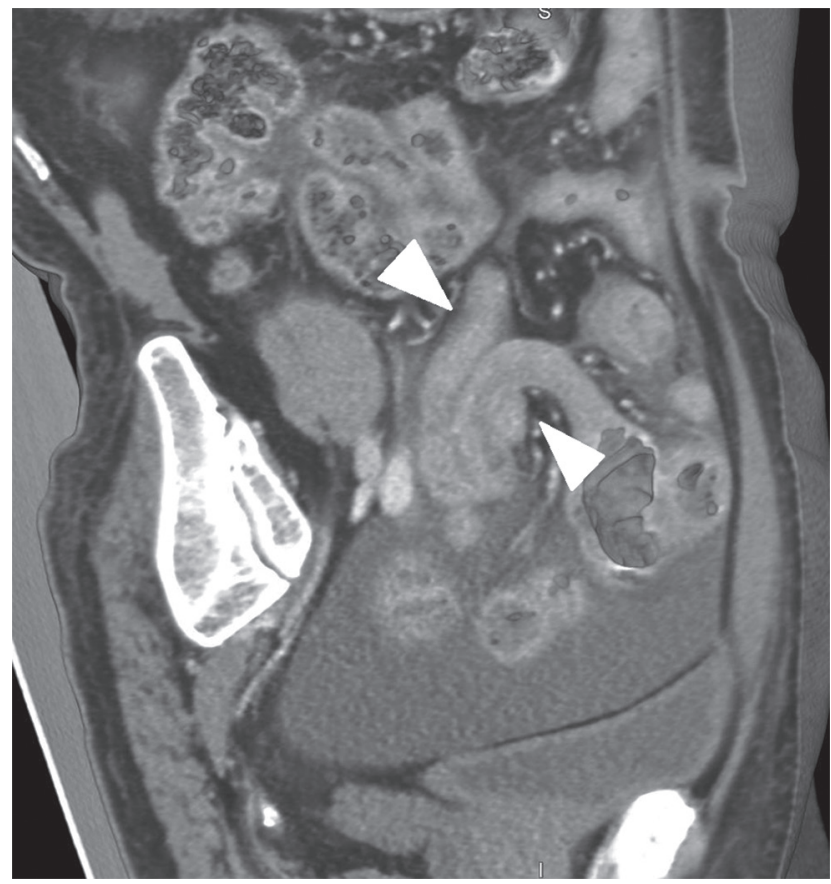

Fig. 4 Closed-loop obstruction. Oblique reformation of contrast-enhanced computed tomography in volume rendered mode shows a closed-loop obstruction with a portion of small bowel passing through the defect (between arrowheads) creating a "beak-like" configuration.

Table 1 When to suspect postoperative internal hernia?

\begin{tabular}{|l|}
\hline History of abdominal surgery \\
\hline Presence of closed loop obstruction \\
\hline Sac-like clustering of bowel \\
\hline Mesenteric "swirl” sign/“swirling mesentery" \\
\hline
\end{tabular}

of small bowel obstruction may not be able to tolerate oral contrast media, and it may not be possible to administer oral contrast media in an emergency setting. Rectal contrast is not useful in the setting of small bowel obstruction. Coronal and sagittal multiplanar reconstructions are necessary to understand the postoperative anatomy. Oblique reconstruction along and perpendicular to the plane of the mesentery of the obstructed small bowel is useful for the delineation of the hernial orifice and complete extent of the involved mesentery ( - Fig. 5). ${ }^{7}$ The use of intravenous contrast is preferred to delineate the anatomy of mesenteric vasculature and to facilitate the identification of decreased bowel wall perfusion and ischemia. Ideally, 1 to $1.5 \mathrm{~mL} / \mathrm{kg}$ of nonionic intravenous iodinated contrast (at least $300 \mathrm{mg} \mathrm{I}_{2} / \mathrm{mL}$ ) is administered at a rate of 3 to $4 \mathrm{~mL} / \mathrm{second}$ with the arterial phase scan obtained at 20 to 25 seconds and venous phase scan at 75 to 90 seconds from the initiation of injection.

\section{Clinical Presentation}

Internal hernias can be completely asymptomatic. Symptomatic patients present with complaints ranging from intermittent vague abdominal discomfort to vomiting and acute abdominal pain. ${ }^{14}$ Patients often present with symptoms of

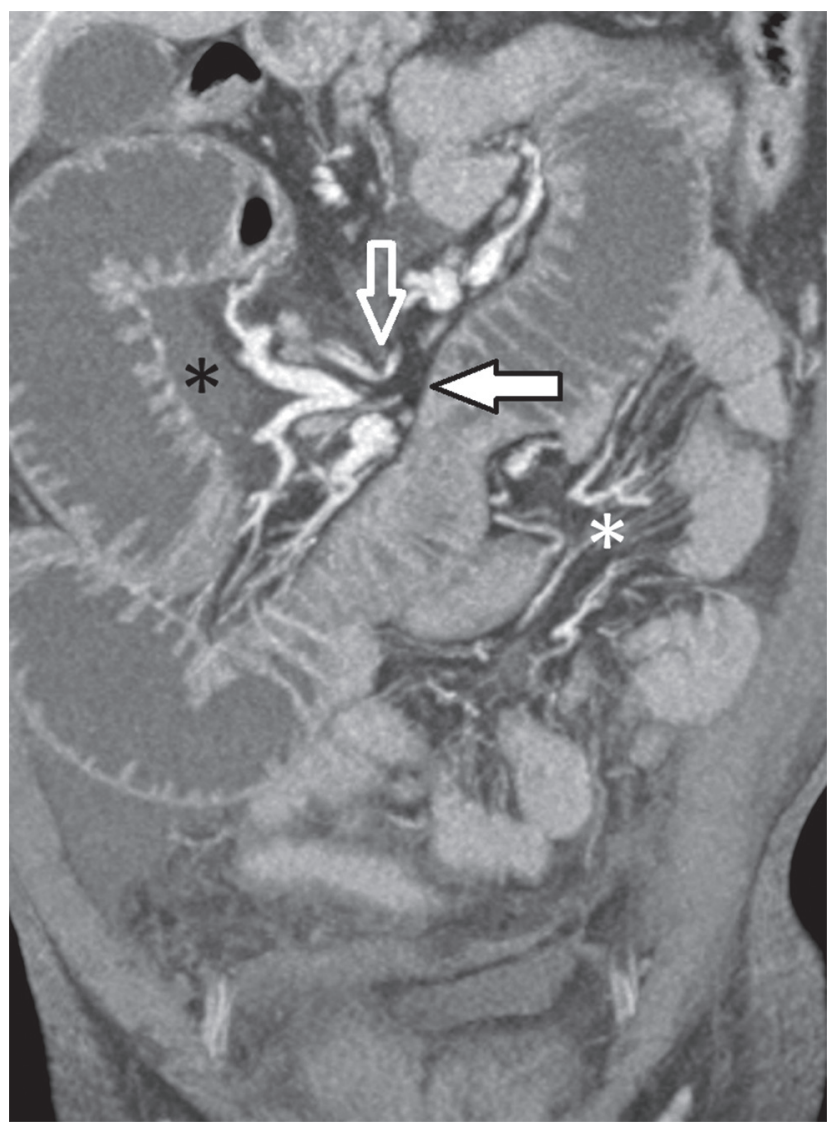

Fig. 5 Localization of the hernial orifice. Coronal reformation of contrast-enhanced computed tomography in maximum intensity projection shows right-sided hernia with closed-loop obstruction. Notice the radial arrangement of the mesenteric vessels that converge at the hernial orifice (solid arrow). Also, note the congested mesenteric veins with a visible kink at the hernial orifice (open arrow). Compare with the mesenteric vessel caliber of the distal collapsed bowel loops (white asterisk). Mesenteric edema is noted in the closed loop (black asterisk).

small bowel obstruction. Time of presentation since surgery is quite variable; occasionally patients present in the immediate postoperative period, but the average time of presentation is 10 to 14 months after surgery. ${ }^{2,14}$ Clinical differential diagnoses include other common postoperative complications such as adhesive bands, ulcers, and anastomotic narrowing.

\section{Classification}

There are three main types of RYGB-associated postoperative internal hernias (-Fig. 6):

- Transverse mesocolonic hernias: This is the most common type of hernia in cases with a retrocolic Roux loop, which extends through the transverse mesocolon.

- Petersen hernias: These hernias course through the potential space between the Roux limb and the transverse mesocolon.

- J-J hernias: In J-J hernia, bowel loops traverse through the small-bowel mesenteric defect at the enteroenterostomy or J-J anastomosis. 


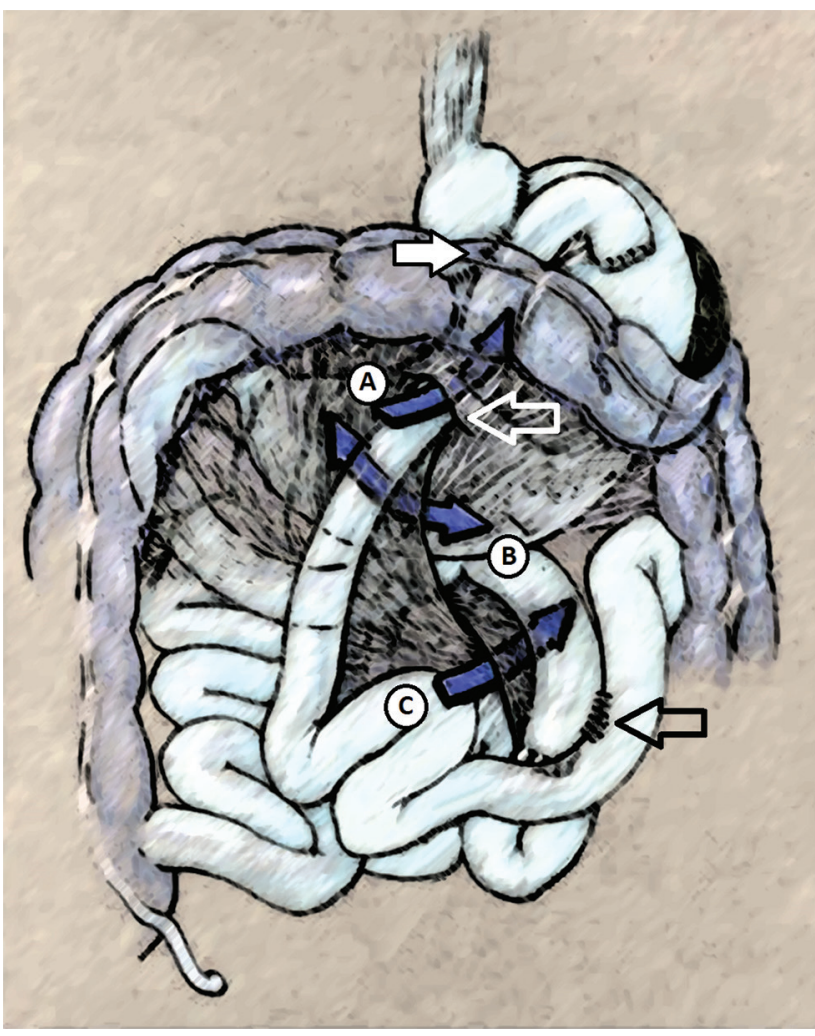

Fig. 6 Schematic diagram of RYGB-related internal hernias. Diagram showing retrocolic RYGB where the Roux (distal or efferent) loop is brought up through the hole created in transverse mesocolon (open white arrow) and gastro-jejunal anastomosis is made (solid arrow). The jejunojejunal anastomosis is most commonly located in the left lower quadrant (open black arrow). Herniation occurring through the hole of in the transverse mesocolon is called the trans-mesocolonic hernia $(\mathbf{A})$, herniation occurring through the potential space behind the Roux loops is called the Petersen hernia (B), and herniation occurring through a gap in the mesenteric anastomosis between the two jejunal loops is called the J-J hernia (C). RYGB, Roux-en-Y gastric bypass.

While the transverse mesocolonic hernia is seen only in patients with a retrocolic Roux limb, the other two hernias are seen in both the retro-and antecolic Roux limb surgeries.

Trans-mesocolonic hernia is the most common postoperative internal hernia overall, particularly in patients with a retrocolic surgical approach. As there is no mesocolonic defect in an antecolic approach, J-J hernias have a higher incidence in this group. $2,7,15$

The incidence of the three subtypes of internal hernias is related to the surgical approach. Open RYGB procedures have a lower incidence of postoperative hernias, attributable to the fixation of bowel loops from peritoneal adhesions associated with open surgical approaches. As mentioned before internal hernia is more common after a laparoscopic surgery as peritoneal adhesions are less common. Therefore, in the laparoscopic approach, intraoperative closure of any potential hernia sites with nonabsorbable sutures and antecolic bypass methods are preferred by some surgeons to decrease the risk of postoperative hernias. ${ }^{14}$ However, internal hernias are still relatively common, potentially related to progressive postoperative loss of mesenteric fat, and resultant enlargement of the mesenteric defect. ${ }^{2}$

\section{Imaging Features}

\section{Key Diagnostic Features ${ }^{6}$}

- Dilated small-bowel loops (maybe pancreaticobiliary, Roux, or distal loops)

- Displacement of the J-J anastomosis out of the left lower or mid-abdomen

- Clustered small-bowel loops often seen in the left upper quadrant (but potentially anywhere in the abdomen)

- Swirling of the mesentery (seen with associated torsion)

- The convergence of bowel loops and vessels with/without associated mesenteric edema

- Dilatation of mesenteric veins in the involved bowel loops due to vascular congestion

In general, the more of the above-mentioned findings are present, the more likely is the diagnosis of internal hernia. ${ }^{6}$

\section{Subtype Specific Features \\ Transmesocolonic hernia}

Herniating bowel loops pass through the defect in the transverse mesocolon created for passage of the retrocolic Roux limb. Thus, herniated loops are typically seen in the left upper quadrant. These hernias account for a large proportion of internal hernias in RYGB patients. The relatively small aperture associated with retrocolic trans-mesocolonic hernia is thought to explain the high risk of torsion and ischemia ( - Fig. 7).

\section{J-J Hernia}

J-J hernias (enteroenterostomy hernias) traverse through the repaired mesenteric defect at the site of the jejunojejunal anastomosis. J-J hernias are the most common type of internal hernia in patients with antecolic RYGB (-Fig. 8). While bowel loops may traverse left-to-right or right-toleft, most commonly the herniated bowel resides in the right upper quadrant. In the case of J-J hernia, the proximal or Y-loop of jejunum is seen stretched around the hernial orifice. The loop may be deformed and displaced from its usual place and, in some cases, may shows torsion or signs of ischemia. $^{7}$

\section{Petersen Hernia}

The Petersen hernia probably is the least common RYGBassociated hernia. Here bowel loops move into the potential space between the transverse mesocolon and the antecolic or retrocolic Roux loop. Petersen hernias may occur alone or in combination with trans-mesocolonic hernias. Herniated bowel loops are more commonly seen in the left upper quadrant. ${ }^{16}$ Unlike the J-J hernia, here the colon and/or the Roux loop of jejunum is seen around the hernial orifice. $^{7}$

\section{Differential Diagnosis Postoperative Leak}

A G-J leak can present with an acute abdomen and tachycardia. Leaks are usually easily seen on upper gastrointestinal fluoroscopy studies but also are readily demonstrated at CT, especially if positive oral contrast is given. 


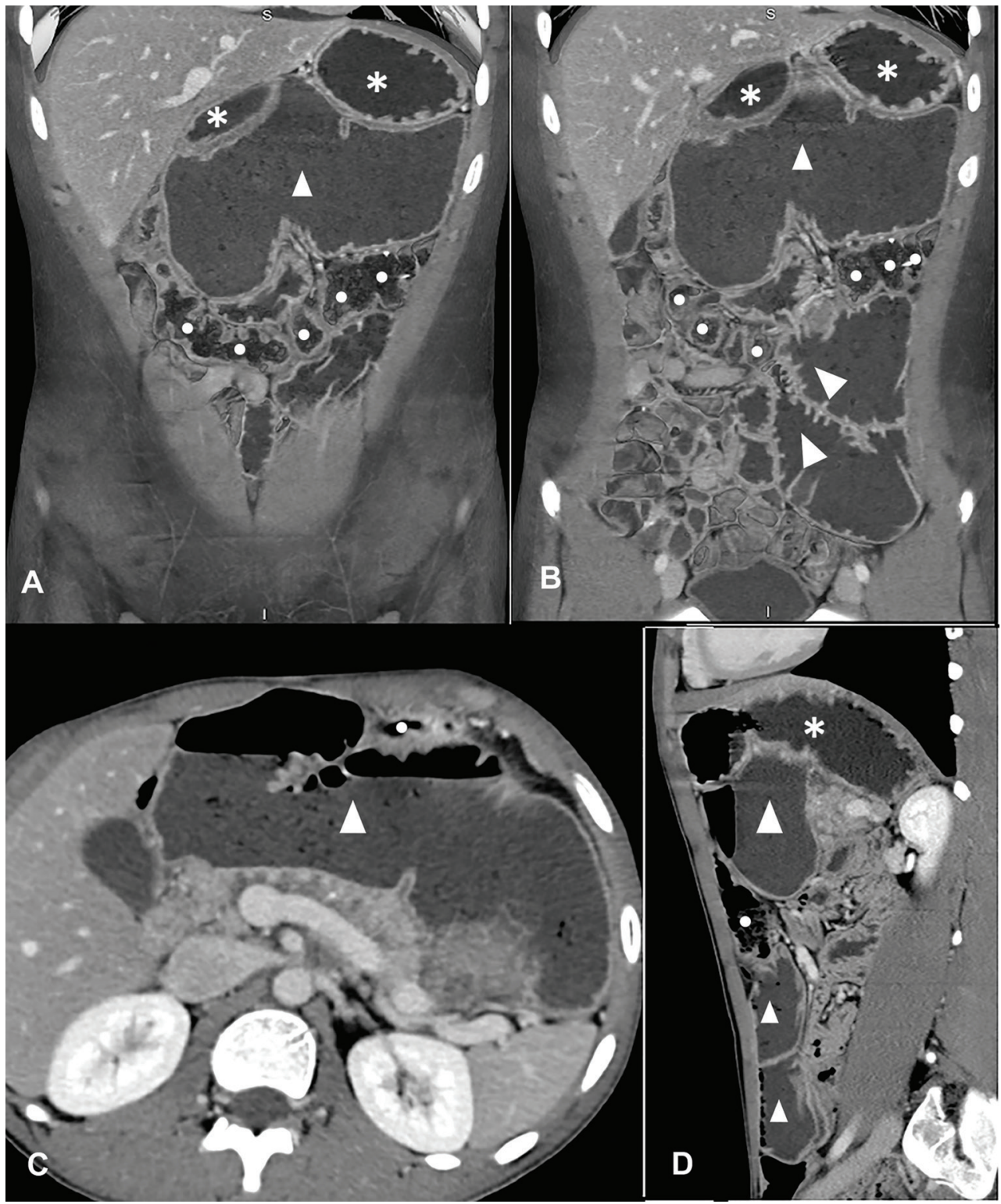

Fig. 7 Transmesocolic hernia after a retrocolic Roux-en-Y gastric bypass surgery. Panel (A) and (B) are volume rendered coronal reconstructions in the venous phase. Panel (A) shows a largely dilated jejunal loop in the region of the lesser sac (triangle) between the stomach (asterisks) and the transverse colon (dots) representing herniation of the jejunum through a defect in the transverse mesocolon. Panel (B) shows the jejunal loops entering the defect (triangles). Panel (C) is an axial section showing the abnormally located dilated herniated jejunum (triangle) posterior to the transverse colon (dot). Panel (D) is a sagittal image showing the herniating jejunal loops (triangles) posterior to the transverse colon (dot) coming to lie inferior to the stomach (asterisk).

\section{Anastomotic Stricture}

Perioperative edema can cause transient anastomotic narrowing but often does not require imaging. Chronic strictures are more common at the G-J than the J-J anastomosis and can result in obstructive symptoms.

\section{Jejunitis and Jejunal Ischemia}

IV contrast-enhanced CT is needed for the diagnosis of mesenteric ischemia. These usually develop in the acute perioperative period.

\section{Intussusceptions}

Intussusceptions typically occur near the J-J anastomosis and may be persistent and obstructive or transient and incidental.

\section{Adhesive Bands}

These are common complications of laparoscopic surgical approaches and mimics internal hernias. Some authors consider band-related obstruction as a fourth type of postoperative internal hernia, but there is no true aperture in such cases. However, the misdiagnosis of adhesive bands as an internal hernia or vice versa is not clinically significant as these have similar complications and clinical course of management (-Fig. 9).

\section{Retro-colic/Retroperitoneal Hernia}

Retroperitoneal hernias can occur through congenital defects in peritoneal fossa, most commonly through the left paraduodenal fossa. ${ }^{17}$ Acquired postoperative retroperitoneal hernias 


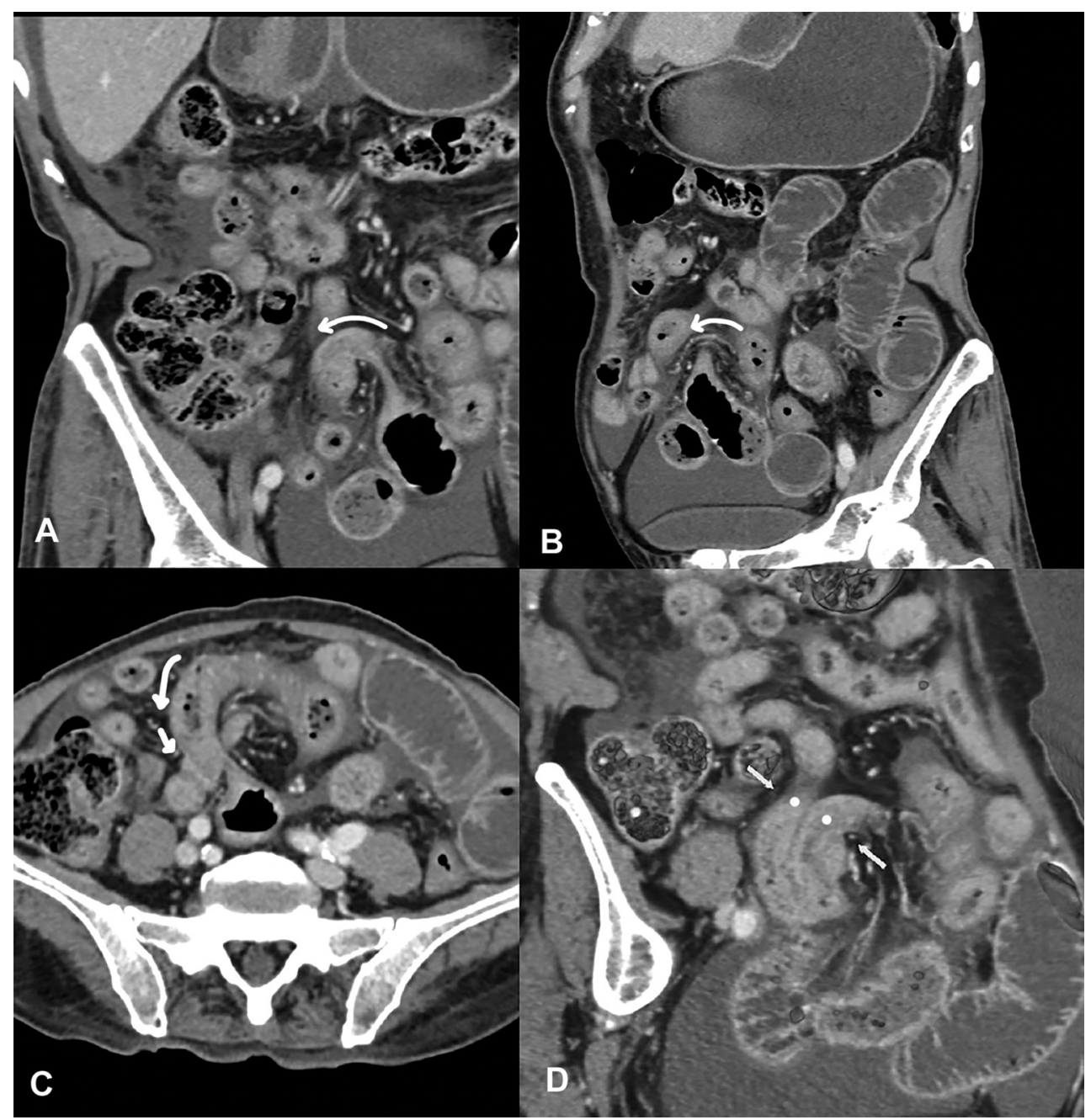

Fig. 8 Entero-enterostomy Hernia. Panels (A) and (B) are oblique coronal computed tomography reconstructions and (C) axial section in the venous phase. Panel (D) is a volume rendered oblique coronal reconstruction through the right iliac fossa in the venous phase. Curved arrows demonstrate a loop of ileum passing through the ileocecal mesentery with a swirl pattern on the axial image. (D) Shows the defect in the mesentery between arrows with the entering and exiting loops (dots).

are rare and are usually associated with iatrogenic or traumatic rent in the peritoneum covering the retroperitoneal structures, most commonly the anterior pararenal fascia and mesentery of the left or right colon. During extraperitoneal nephrectomy where the left or right colon is mobilized medially, a rent may be created in colonic mesentery inadvertently. If that is not recognized or repaired, small bowel may herniate through this defect into the potential space in retroperitoneum. In such cases, a cluster of small bowels will be seen lateral to the right or left colon, and the mesentery will

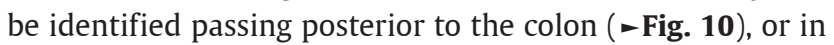
some cases of right-sided hernia, posterior to the second portion of duodenum ( - Fig. 11). These hernias can occur in up to $0.5 \%$ of cases of laparoscopic donor nephrectomy..$^{18}$ These are also described as a complication of abdominoperineal resection (APR), ${ }^{17}$ radical nephrectomy, ${ }^{19}$ or pelvic trauma. ${ }^{20}$

\section{Perineal Hernia}

Perineal hernia $(\mathrm{PH})$ is defined as the herniation of abdominal viscera through a pelvic floor defect. Primary PH is usually due to long-standing ascites, obesity or pregnancy, ${ }^{21}$ and rarely congenital. ${ }^{22}$ Secondary $\mathrm{PH}$ is usually postoperative and can occur after pelvic exenteration, APR or extra-levator abdominoperineal excision (ELAPE). PH can be classified as anterior or posterior, depending on its relationship to the transverse perineal muscle. ${ }^{21}$ Primary PHs are more common in women and the anterior PHs occur almost exclusively in women. ${ }^{23}$ No gender predilection is described in secondary PHs. ${ }^{24}$ The incidence of posterior PH has been reported to be 1 to $13 \%$ after abdominoperineal excision (APE) ${ }^{25}$ and up to $26 \%$ after ELAPE. ${ }^{26}$ However, the incidence of symptomatic PH is less than $1 \%$. The incidence of $\mathrm{PH}$ cases seems to be increasing. ${ }^{27}$ This may be due to increased use of ELAPE in the treatment of rectal cancer, as it involves a more extensive excision of the perineum than APE does. It may also be related to the use of neoadjuvant radiotherapy in rectal cancer that impairs wound healing and possibly predisposes to $\mathrm{PH}$.

$\mathrm{PH}$ is usually a late complication of APR with a median interval of 10 months. ${ }^{28}$ Clinical features include a bulge in the perineum or gluteal region with/without pain or 

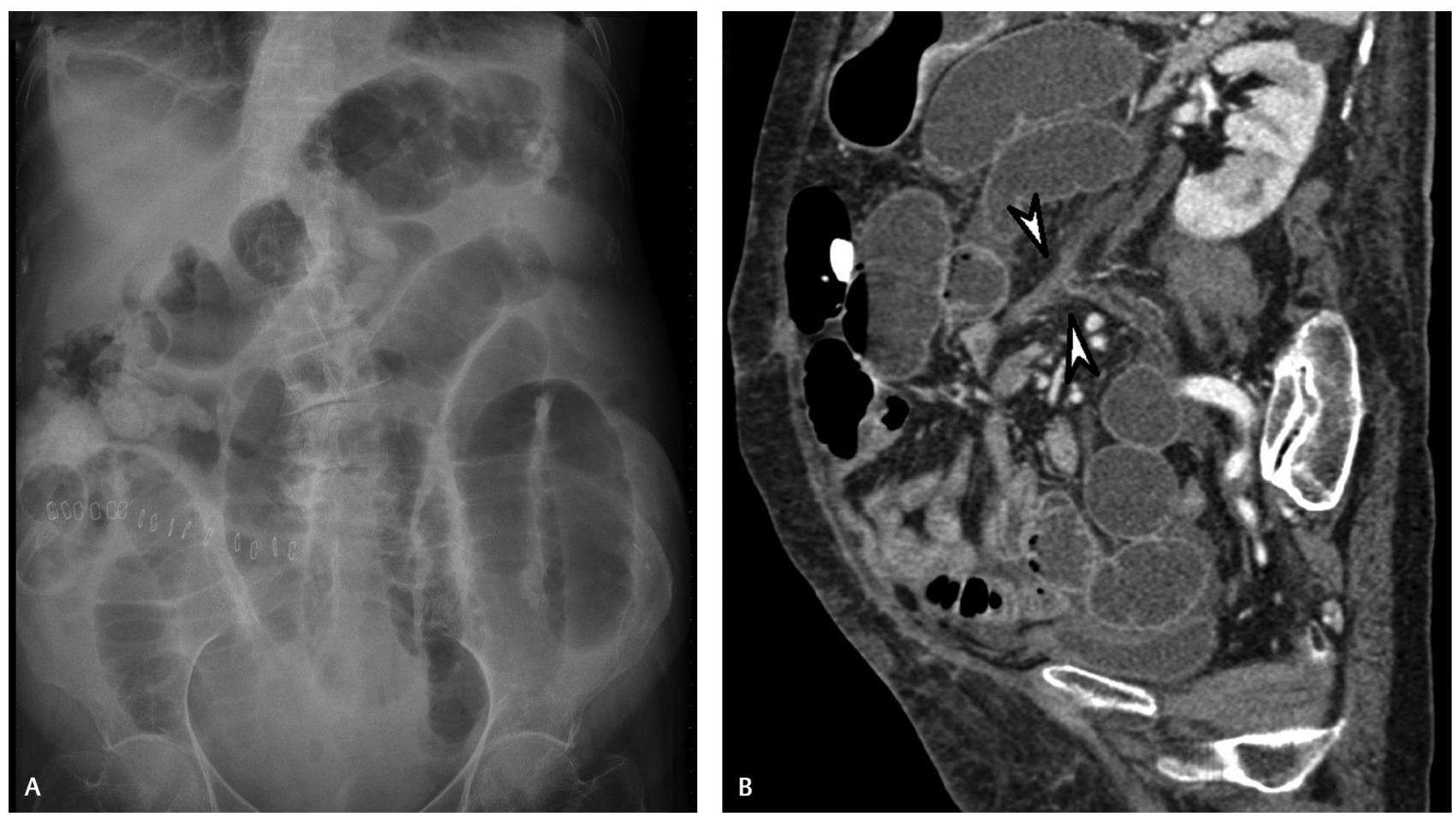

Fig. 9 Small bowel obstruction from postoperative band. (A) Supine abdominal radiograph (anteroposterior view) shows dilated small bowel loops filling up the central abdomen. Large bowel loops are collapsed with no rectal gas shadow suggesting small bowel obstruction. Notice surgical staples in the right lower abdomen from previous surgical exploration. (B) Sagittal reformation of contrast-enhanced computed tomography shows a Y-shaped band (between arrowheads) at the site of the transition of small bowel caliber. The adhesive band was confirmed at surgery.
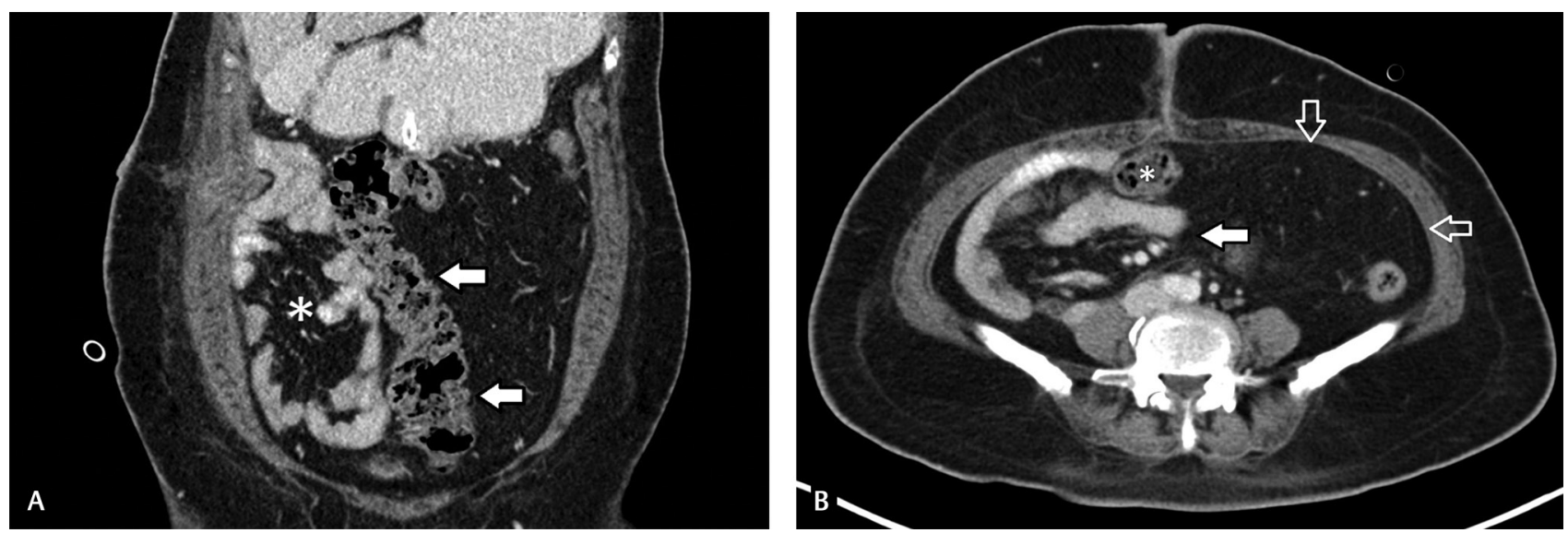

Fig. 10 Retro-colic retroperitoneal hernia in a postnephrectomy patient: (A) coronal reformat of contrast-enhanced CT shows an abnormal position of small bowel loops (asterisk) lateral to the ascending colon (arrows). (B) Axial contrast-enhanced CT shows small bowel mesentery (solid arrow) passing posterior to the ascending colon (asterisk). Notice the fatty greater omentum is bunched up in the left side of the abdomen (open arrows). CT, computed tomography.

discomfort, intestinal obstruction or urinary symptoms depending upon the site and extent of herniation. CT can demonstrate the herniated content and neck of the hernia sac. Strangulation is uncommon. ${ }^{21}$ The presence of herniated content below the level of levator ani muscle indicates a PH (-Fig. 12). The level of levator ani muscle can be ascertained on sagittal reconstruction of $\mathrm{CT}$ through lower abdomen and pelvis by drawing an imaginary line from inferior border of symphysis pubis to last coccygeal joint. Levator ani muscle may not be identifiable in all cases.
In some cases, the bowel loops can be identified in ischiorectal fossa or perineum. Some authors suggest performing the $\mathrm{CT}$ with the Valsalva maneuver to demonstrate the hernia.

\section{External Hernia}

Postoperative external hernia is defined by an outpouching of bowel loops and other viscera through defects in the abdominal wall that were created during or occurred as a complication of surgery. 


\section{Incisional Hernia}

An incisional hernia occurs through the site of the incision of previous surgery. Incisional hernias of the abdominal wall can be divided into anterior and lateral abdominal wall hernias based on the position of the defect relative to linea semilunaris. Defects that occur between the two lineae semilunaris are called central anterior abdominal wall hernia and defects that occur through the space between linea semilunaris and posterior paraspinal muscles are called lateral abdominal wall hernia (-Fig. 13). ${ }^{29}$ The incidence of anterior abdominal wall incisional hernia varies from 5 to $15 \%$ after

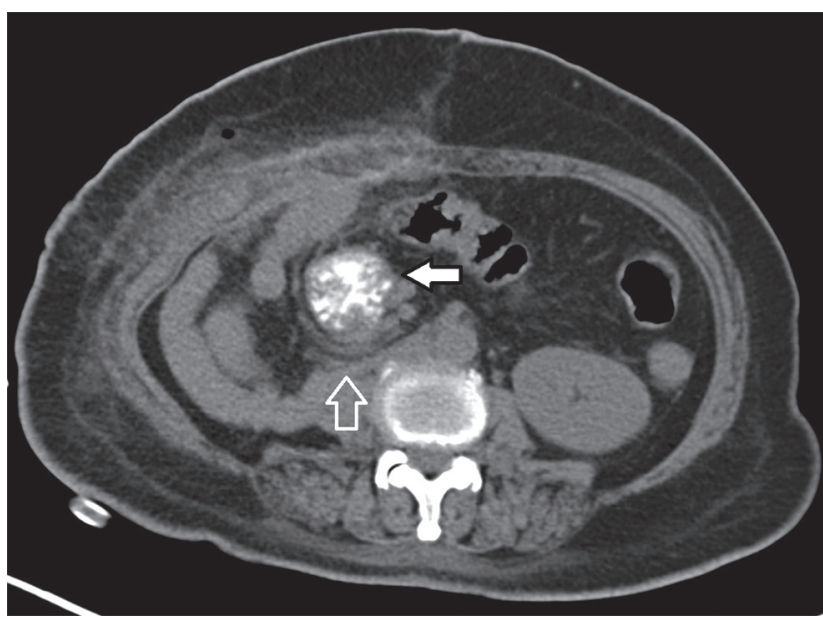

Fig. 11 Retro-duodenal retroperitoneal hernia in postnephrectomy patient: axial noncontrast computed tomography with oral contrast shows oral contrast in the second portion of the duodenum (solid arrow). Small bowel loops with mesentery (open arrow) are seen passing posterior to the duodenum. Notice the lack of passage of oral contrast to distal bowel loops. open surgical procedures and 1 to $3 \%$ in patients after minimally invasive surgical procedures. ${ }^{30}$ It is more common after colorectal and vascular surgeries ${ }^{31}$ and more common with midline incisions than that with transverse or off-midline incisions. ${ }^{32}$ Other risk factors include obesity, smoking, and previous abdominal surgeries. ${ }^{31,33}$ Other than the possibility of obstruction and ischemia, giant incisional hernias are complicated by skin ulceration, infections, and enterocutaneous fistula. Giant incisional hernias are variably defined as hernias with abdominal wall defect size larger than 10 to $15 \mathrm{~cm}$ or a hernial sac size larger than 100 to $225 \mathrm{cc}$.

CT for the evaluation of anterior abdominal wall hernia should be done after administration of intravenous contrast media and with and without Valsalva maneuver. ${ }^{34}$ The important preoperative information that must be provided is given in - Table $\mathbf{2}$. The feasibility of a repair of the defect is determined by several factors that can be demonstrated in preoperative imaging. These include the following: (1) size of the defect relative to the overall size of the ventral abdominal wall, (2) size of sac relative to the intra-abdominal volume, and (3) amount of visceral fat. The component separation index gives an estimate of the size of the defect relative to the size of the abdominal wall and can easily be calculated on axial CT ( - Fig. 14). Christy et al showed that patients who needed an interpositional mesh repair of incisional hernia had a higher CT separation index (CSI) $(0.21 \pm 0.04)$ than those who did not $(0.11 \pm 0.06)$. The second group also had a lower hernia recurrence rate. The authors concluded that a higher CSI usually indicates a need for mesh placement and a higher postoperative recurrence rate. ${ }^{35} \mathrm{~A}$ large sac size relative to the preoperative intra-abdominal volume indicates the possibility of high intra-abdominal pressure after operative reduction and the consequent risk of failure. CT-based
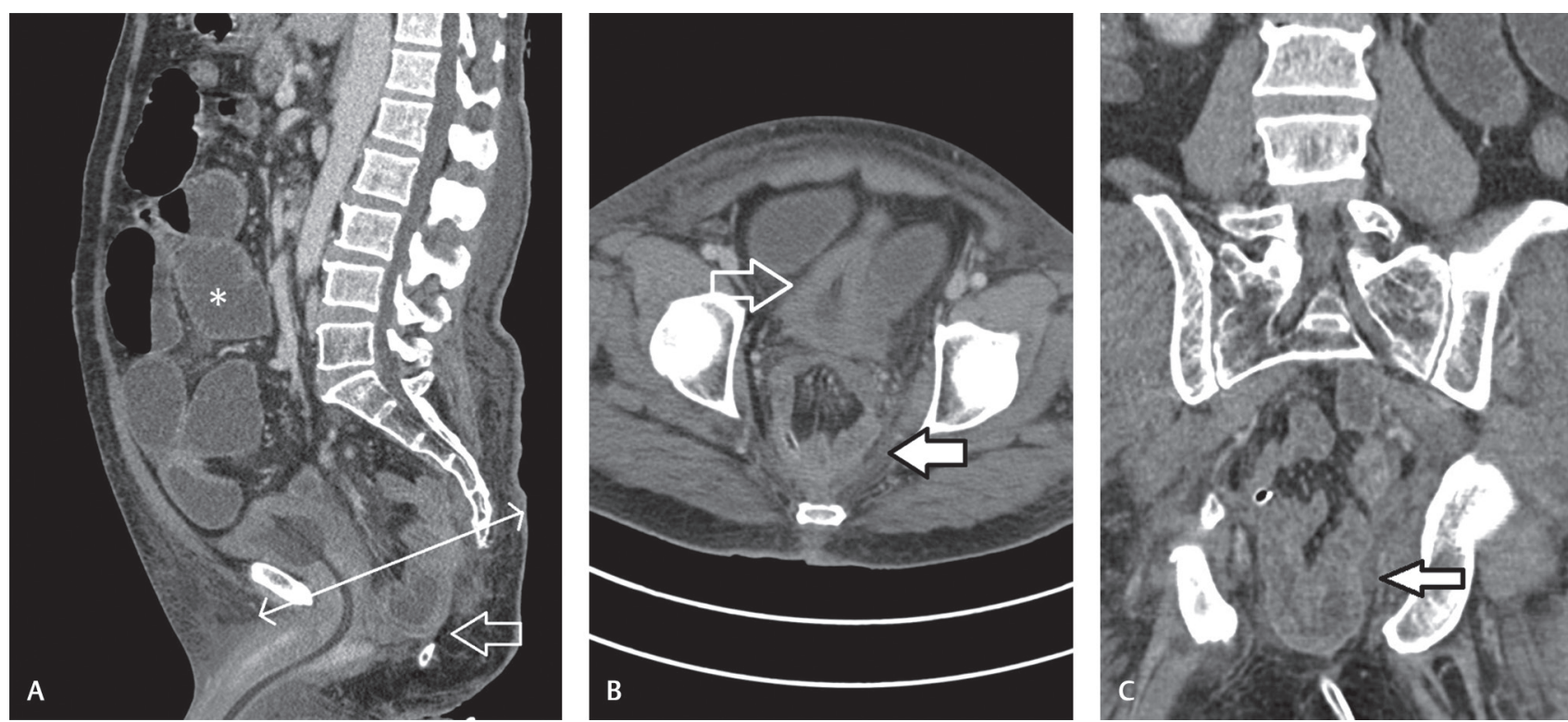

Fig. 12 Perineal hernia in a patient after extra-levator abdominoperineal resection. (A) Sagittal reformat of contrast-enhanced CT shows presence of small bowel loops (open arrow) inferior to the line of levator ani muscle (double-headed arrow). Notice dilated small bowel loops (asterisk). (B) Axial contrast-enhanced CT shows presence of small bowel and mesentery (solid arrow) posterior to the urinary bladder (open arrow). (C) Coronal contrast-enhanced CT shows herniating small bowel loops through the levator sling (arrow). Levator muscle is often not identified in such cases. CT, computed tomography. 


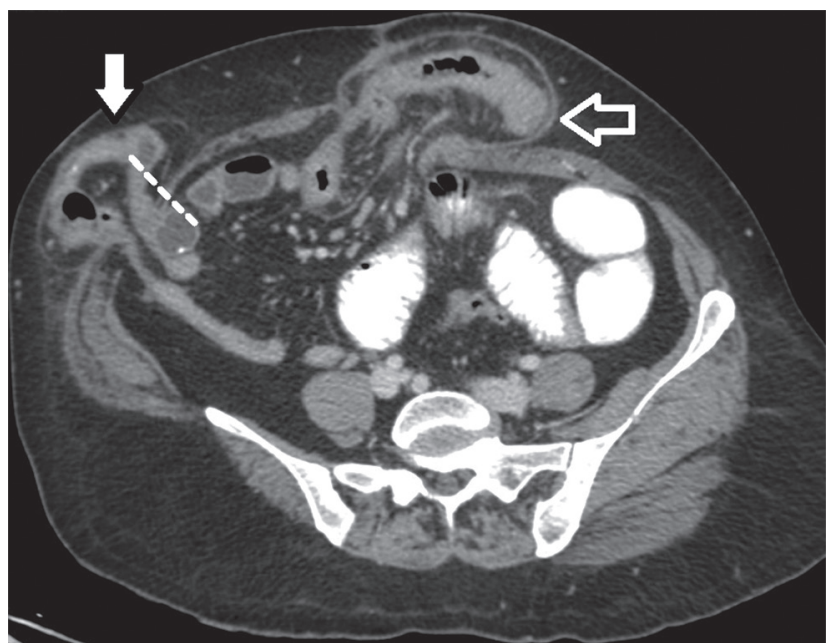

Fig. 13 Anterior and lateral abdominal wall incisional hernia in a patient status postlaparotomy. Axial contrast-enhanced computed tomography shows herniation of small bowel loops between the two recti muscles (open arrow, anterior hernia) and lateral to the right rectus muscle (solid arrow, lateral hernia). The linea semilunaris is marked with dashed line.

Table 2 Imaging evaluation in postoperative ventral hernia

\begin{tabular}{|l|}
\hline - Size of the defect (both transverse and longitudinal) \\
\hline - Content of the hernia \\
\hline - Presence of omental coverage \\
\hline - The thickness of abdominal wall musculature \\
\hline - Presence or absence of fatty atrophy of abdominal wall \\
muscles \\
\hline - Signs of previous surgery (adhesion, fluid collection, other \\
smaller defects) \\
\hline - Presence of mesh \\
\hline - Valsalva: mobility of the hernial content and distensibility \\
of abdominal wall muscles \\
\hline
\end{tabular}

morphometric analysis includes automated algorithms for the calculation of subcutaneous fat area (in $\mathrm{cm}^{2}$ ), total body area (in $\mathrm{cm}^{2}$ ), and total body circumference (in $\mathrm{cm}$ ). Levi et al showed that in a group of patients who underwent mesh repair of incisional hernia patients who develop surgical site infection and dehiscence showed significantly higher subcutaneous fat area, total body area, and total body circumference; while there is no significant difference detected between the groups in terms of body mass index or the type of mesh used. This suggests CT-based morphometric analysis can offer more detailed analysis of body composition than simple body weight measurement that can help in risk stratification of postoperative infection and dehiscence after hernia repair. ${ }^{36}$

Clinical differential diagnoses of ventral hernia include abdominal wall tumors, rectus hematoma, fluid collection within anterior abdominal wall, and eventration. ${ }^{30,37} \mathrm{CT}$ is also useful in the detection of complications of incisional hernia such as intestinal obstruction and ischemic changes in bowel ( - Fig. 15).

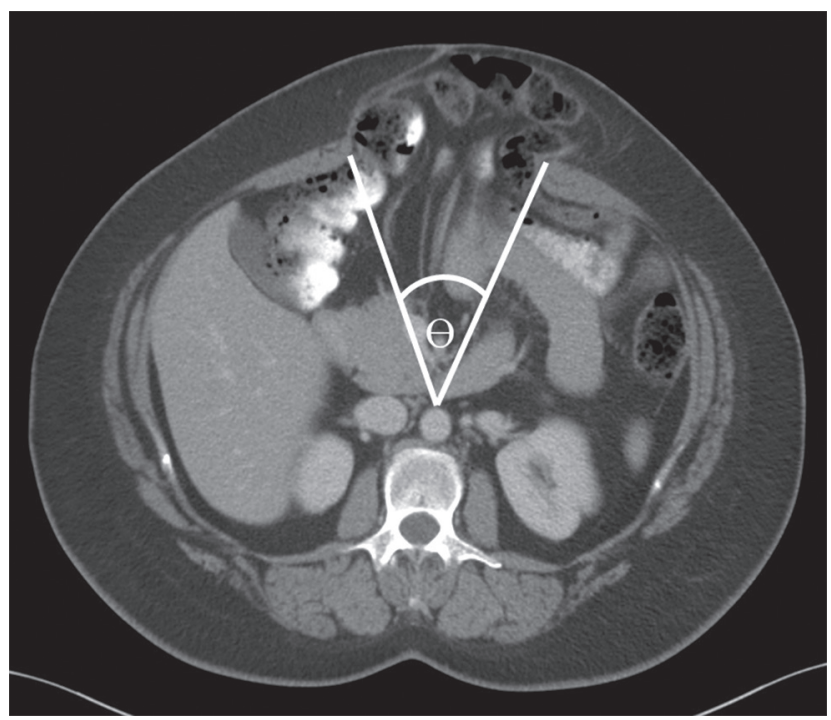

Fig. 14 Component separation index. Axial contrast-enhanced computed tomography shows an anterior incisional hernia with separation of recti (diastasis). An angle is drawn between the lines joining the medial margin of recti (or, the margin of the hernial orifice) at the widest portion of the hernia and a fixed point within the abdomen, most commonly the $\operatorname{aorta}(\Theta)$. The component separation index is given by $\Theta / 360$.

\section{Parastomal Hernia}

Parastomal hernias are often regarded as a subtype of the incisional hernia. ${ }^{37}$ It is defined by the herniation of the intra-abdominal content through a defect in the vicinity of the stoma. These can occur in up to 10 to $56 \%$ of end colostomies. ${ }^{38}$ The incidence of parastomal hernias is less after ileostomy. ${ }^{39}$ However, these hernias are symptomatic in only approximately $10 \%$ of cases, ${ }^{40}$ although CT can pick up a parastomal hernia in up to $78 \%$ cases..$^{41} \mathrm{~A}$ stoma can be brought out in the anterior abdominal wall through the rectus abdominis muscle (trans-rectus) or lateral to it (para-rectus). Some researchers have suggested that the incidence of parastomal hernia is more common in the pararectus approach; $4^{2}$ however, others found no significant difference. ${ }^{41,43} \mathrm{~A}$ parastomal hernia is often clinically invisible. CT helps in the detection of hernia (-Fig. 16) and defines its position which is more commonly lateral to the stoma than medial to it. Imaging also helps to define the size and content of the hernia along with the presence of any complication ( - Fig. 17). Clinically a stomal hernia may be confused with other complications such as stomal prolapse, parastomal collection, and abscess formation (- Fig. 18).

\section{Port Site Hernia}

Port site hernia or trocar site hernia is a special variant of incisional hernia that occurs through the laparoscopic trocar insertion site. Although the incidence of true port site hernia is low, it is shown to be underestimated by surgeons and can range from as low as $0.74 \%$ to as high as $39.3 \%{ }^{44}$ The incidence is highest after laparoscopic colorectal surgery. ${ }^{45}$ Due to the small size of the neck, port site hernia has a particularly high risk of obstruction and strangulation ${ }^{44,45}$ ( - Fig. 19). A larger trocar size is related to a higher incidence of hernia, ${ }^{46}$ therefore it is recommended that a fascial defect larger than 

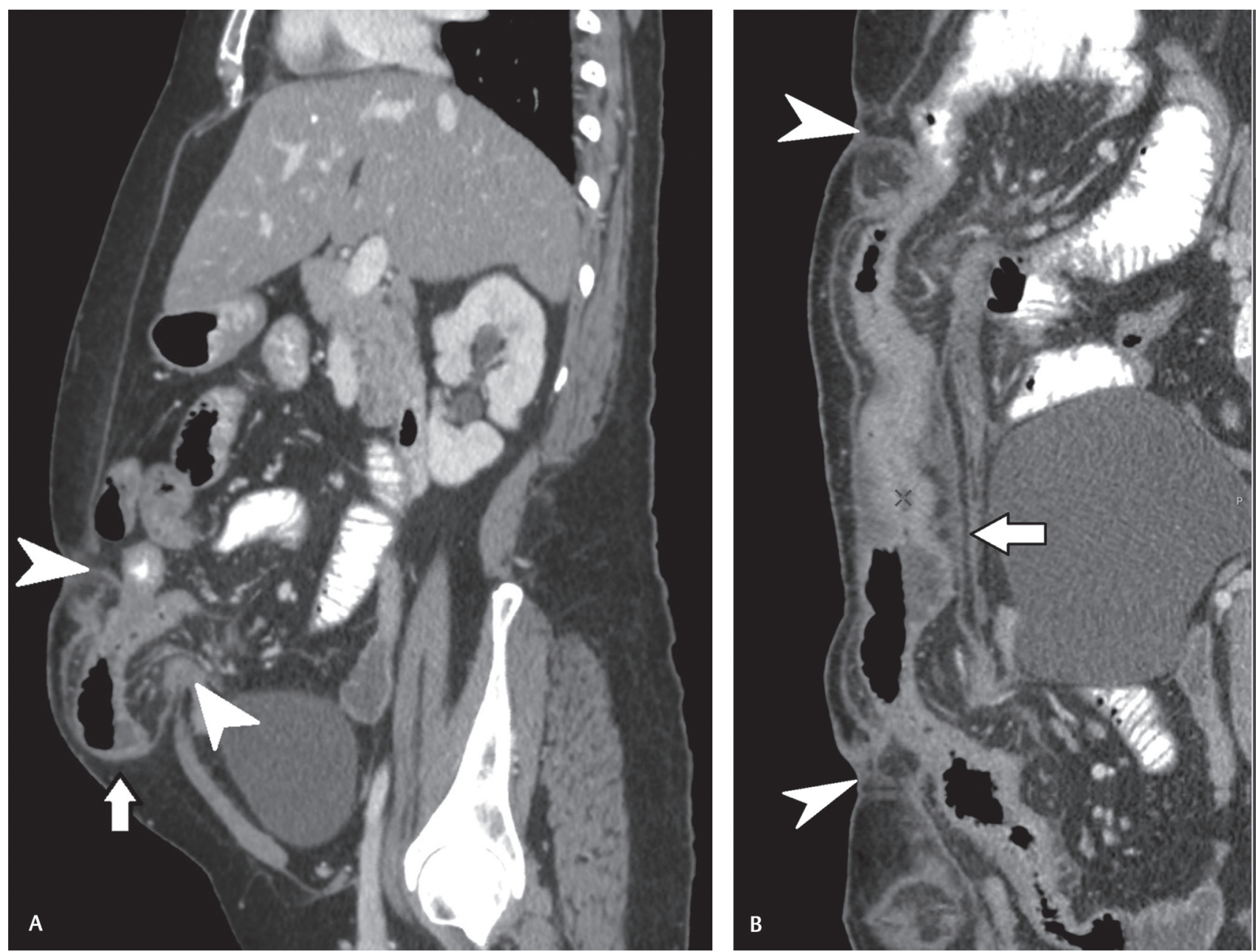

Fig. 15 Incisional hernia with obstruction. (A) Sagittal reformat of contrast-enhanced computed tomography shows midline ventral incisional hernia with the neck of the hernial sac marked by arrowheads. (B) Curved multiplanar reconstruction of the same image. Bowel loop within the hernia sac is unfolded by tracing the curve of the bowel. The neck of the sac, that is, the beginning and the end of the loop, is again marked by arrowheads. Notice the sudden change of caliber of the small bowel as the loop enters the sac (superior arrowhead). There is a lack of passage of oral contrast within the sac associated with wall thickening of the closed-loops and mesenteric edema (arrow).

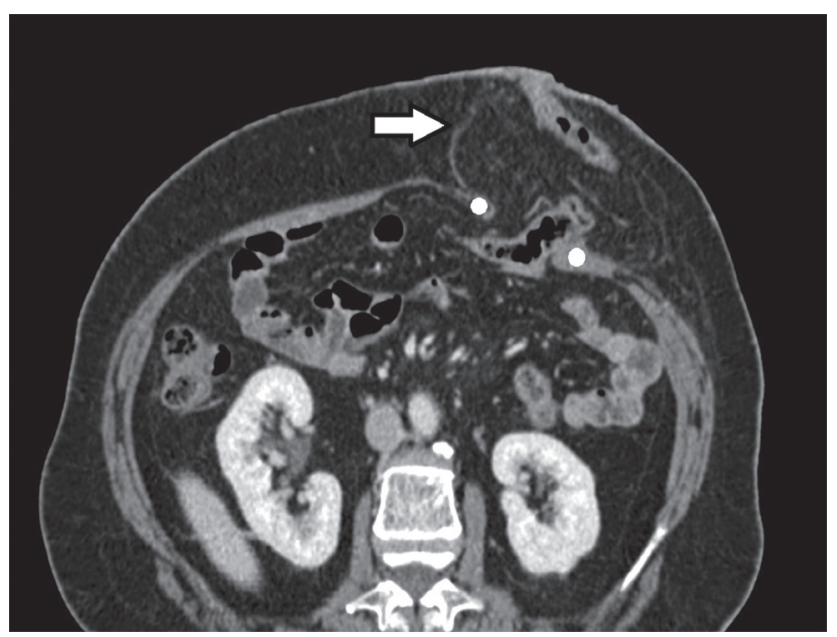

Fig. 16 Clinically invisible colostomy site parastomal hernia. Oblique axial contrast-enhanced computed tomography shows left trans-rectus sigmoid colostomy (left rectus muscle is marked by dots). Omental fat has herniated through the stoma defect (arrow). Notice the hernia has not produced any abdominal wall swelling or obstruction, and hence, it was clinically silent.
$10 \mathrm{~mm}$ should be closed with peritoneum. ${ }^{45}$ In the early postoperative period, a port site dehiscence may occur where there is a separation of the fascia with intact overlying skin. A hernia sac usually forms 30 days after the surgery. ${ }^{47}$ Therefore, trocar site hernia can be classified into three types. In the early-onset type, there is fascial dehiscence with intact skin and no peritoneal sac is formed. This is often associated with small-bowel obstruction. In the late-onset type, a peritoneal sac is formed and skin is still intact. Small-bowel obstruction is less common in this type of hernia. In the third type, the skin is also dehiscent and protruding structures can be seen. This type is also called port site evisceration..$^{47,48}$ Following oncological surgery, especially in presence of intraperitoneal malignancy and ascites, a port site metastasis may occur, which should be differentiated from a port site hernia.

\section{Conclusion}

Postoperative internal hernia should always be considered in the differential diagnosis of acute abdomen in patients with 


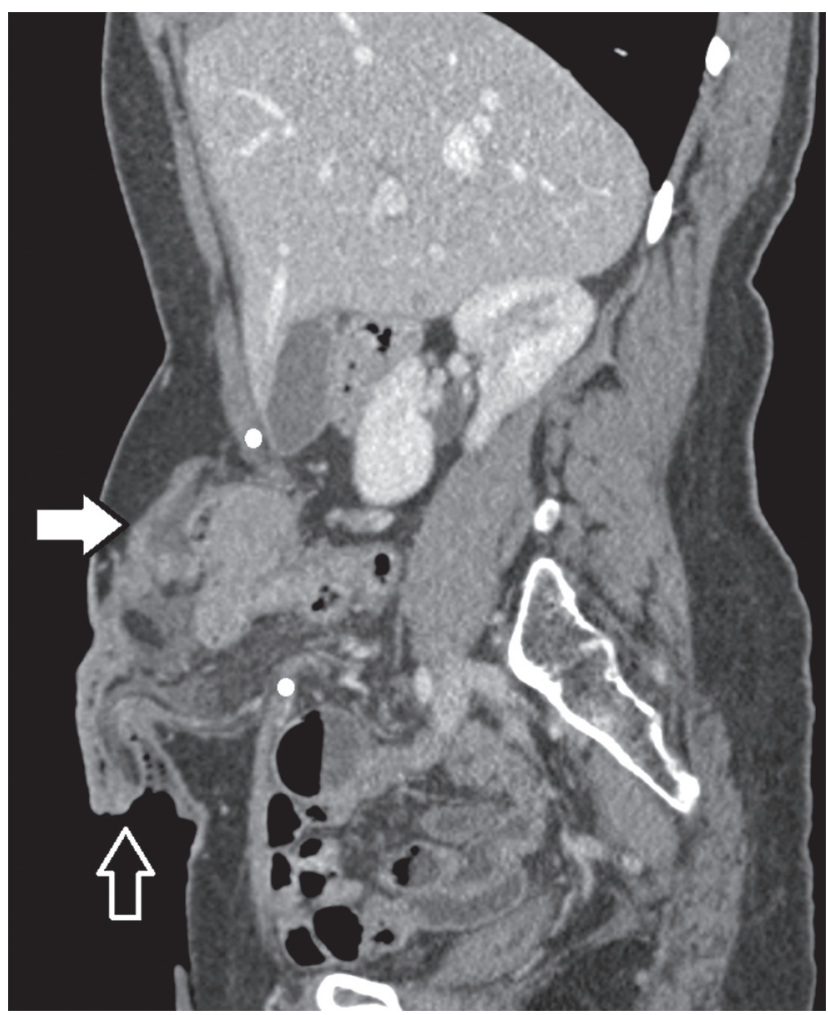

Fig. 17 Ileostomy site parastomal hernia with stomal prolapse and incarceration. Sagittal reformat of contrast-enhanced computed tomography shows right-sided ileostomy with right rectus abdominis muscle marked by dots. Notice that the stoma has prolapsed (open arrow). The hernial sac (solid arrow) contains edematous small bowel loops with mesenteric fluid collection. a recent or known history of operation. It is particularly common in patients undergoing laparoscopic RYGB surgery. CT with IV contrast is the investigation of choice. Cardinal signs of internal hernia such as closed-loop obstruction, swirling of mesentery, and sac-like clustering of small bowel should be searched for. Postoperative external hernias are usually

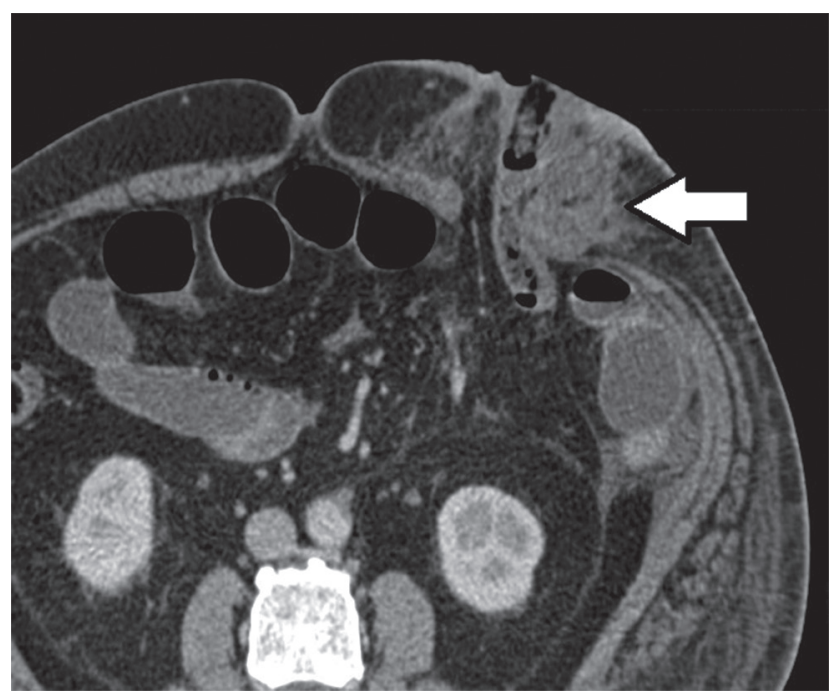

Fig. 18 Colostomy site abscess. The patient has presented with local swelling and redness involving the area of colostomy. Axial contrast-enhanced computed tomography shows left sided trans-rectus sigmoid colostomy with fluid collection and fat stranding around the colostomy site (arrow). No herniation of bowel or omental fat is noted. Aspiration yielded pus.
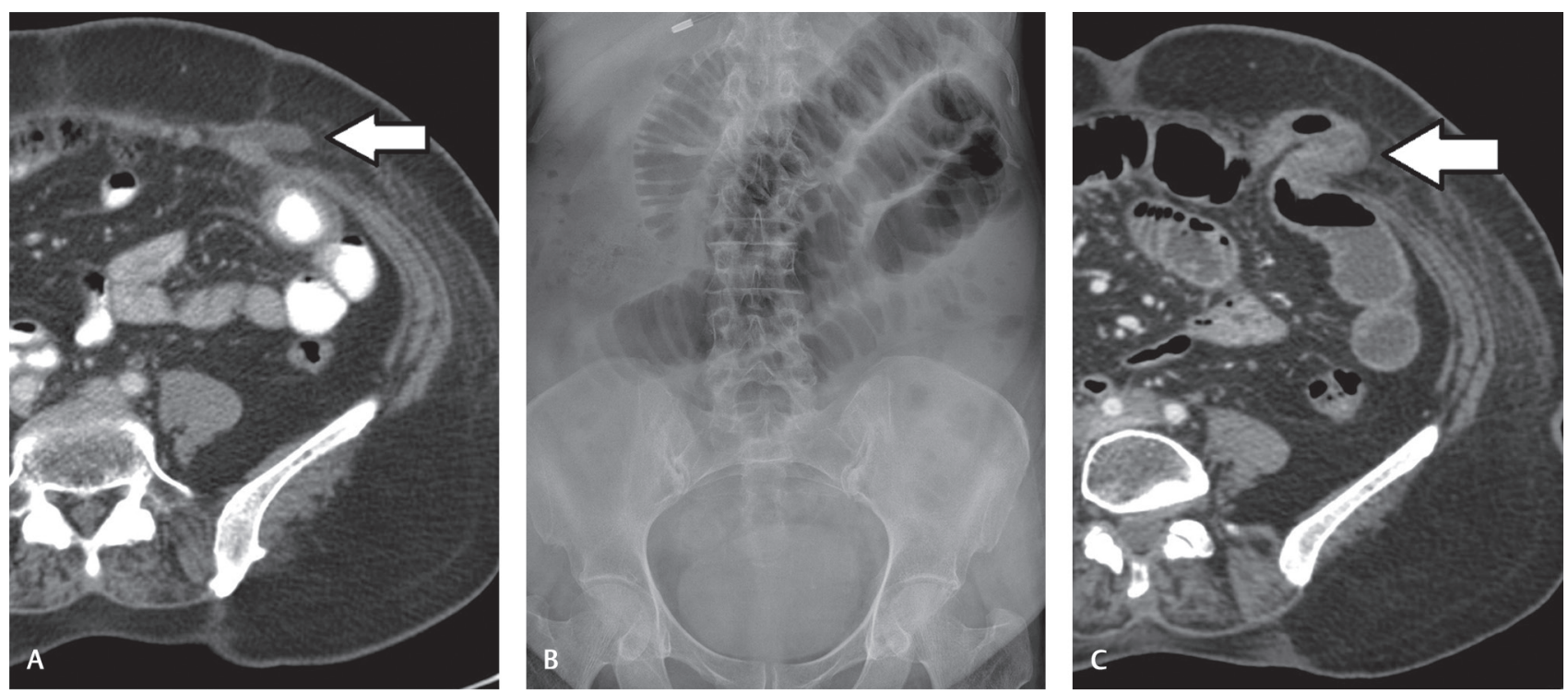

Fig. 19 Port site hernia with obstruction. (A) Axial contrast-enhanced CT 3 weeks after laparoscopic surgery shows small peritoneal outpouching containing fluid through the left paramidline port (arrow). This finding was ignored. (B) Total 2 weeks later, the patient presented to the emergency with acute abdominal pain and features of intestinal obstruction. Supine abdominal radiograph shows dilated jejunal loop. (C) Axial contrast-enhanced CT shows herniation of small loop through the port site causing small bowel obstruction. CT, computed tomography. 
clinically evident. However, CT helps in delineation and surgical planning as well as in ruling out complications and other differential diagnoses.

\section{Funding}

None.

\section{Conflict of Interest}

None declared.

\section{References}

1 Johnson EE, Simpson AN, Harvey JB, Simpson KN. Bariatric surgery implementation trends in the USA from 2002 to 2012. Implement Sci 2016;11(1):21

2 Ahmed AR, Rickards G, Husain S, Johnson J, Boss T, O'Malley W. Trends in internal hernia incidence after laparoscopic Rouxen-Y gastric bypass. Obes Surg 2007;17(12):1563-1566

3 Garza E Jr, Kuhn J, Arnold D, Nicholson W, Reddy S, McCarty T. Internal hernias after laparoscopic Roux-en-Y gastric bypass. Am J Surg 2004;188(6):796-800

4 Karila-Cohen P, Cuccioli F, Tammaro P, et al. Contribution of computed tomographic imaging to the management of acute abdominal pain after gastric bypass: correlation between radiological and surgical findings. Obes Surg 2017;27(8):1961-1972

5 Carucci LR, Turner MA, Shaylor SD. Internal hernia following Roux-en-Y gastric bypass surgery for morbid obesity: evaluation of radiographic findings at small-bowel examination. Radiology 2009;251(3):762-770

6 Lockhart ME, Tessler FN, Canon CL, et al. Internal hernia after gastric bypass: sensitivity and specificity of seven $\mathrm{CT}$ signs with surgical correlation and controls. AJR Am J Roentgenol 2007;188(3):745-750

7 Hongo N, Mori H, Matsumoto S, Okino Y, Takaji R, Komatsu E. Internal hernias after abdominal surgeries: MDCT features. Abdom Imaging 2011;36(4):349-362

8 Furukawa A, Yamasaki M, Furuichi K, et al. Helical CT in the diagnosis of small bowel obstruction. Radiographics 2001;21(2):341-355

9 Doishita S, Takeshita T, Uchima Y, et al. Internal hernias in the era of multidetector CT: Correlation of imaging and surgical findings. Radiographics 2016;36(1):88-106

10 Levine MS, Carucci LR. Imaging of bariatric surgery: normal anatomy and postoperative complications. Radiology 2014;270(2):327-341

11 Fridman A, Moon R, Cozacov Y, et al. Procedure-related morbidity in bariatric surgery: a retrospective short- and midterm follow-up of a single institution of the American College of Surgeons Bariatric Surgery Centers of Excellence. J Am Coll Surg 2013;217(4):614-620

12 Scheirey CD, Scholz FJ, Shah PC, Brams DM, Wong BB, Pedrosa M. Radiology of the laparoscopic Roux-en-Y gastric bypass procedure: conceptualization and precise interpretation of results. Radiographics 2006;26(5):1355-1371

13 Ahmed AR, Rickards G, Johnson J, Boss T, O'Malley W. Radiological findings in symptomatic internal hernias after laparoscopic gastric bypass. Obes Surg 2009;19(11):1530-1535

14 Higa KD, Ho T, Boone KB. Internal hernias after laparoscopic Roux-en-Y gastric bypass: incidence, treatment and prevention. Obes Surg 2003;13(3):350-354

15 Iannuccilli JD, Grand D, Murphy BL, Evangelista P, Roye GD, Mayo-Smith W. Sensitivity and specificity of eight CT signs in the preoperative diagnosis of internal mesenteric hernia following Roux-en-Y gastric bypass surgery. Clin Radiol 2009;64(4):373-380
16 Ximenes MAS, Baroni RH, Trindade RMC, et al. Petersen's hernia as a complication of bariatric surgery: CT findings. Abdom Imaging 2011;36(2):126-129

17 Alkhoury F, Martin J. Case report: complex retroperitoneal herniation after abdominoperineal resection. Int J Surg Case Rep 2012;3(12):584-586

18 Regan JP, Cho ES, Flowers JL. Small bowel obstruction after laparoscopic donor nephrectomy. Surg Endosc 2003;17(1): 108-110

19 Milosevic I, Dorion H, Ricchiuti D. Incarcerated retroperitoneal hernia following total extraperitoneal laparoscopic radical nephrectomy. JSLS 2011;15(3):424-426

20 Stubbart JR, Merkley M. Bowel entrapment within pelvic fractures: a case report and review of the literature. J Orthop Trauma 1999;13(2):145-148

21 Lubat E, Gordon RB, Birnbaum BA, Megibow AJ. CT diagnosis of posterior perineal hernia. AJR Am J Roentgenol 1990; 154(4):761-762

22 Upreti L, Kumar A, Upreti L. Congenital perineal hernia : radiographic findings and role of imaging in diagnosis. Indian J Radiol Imaging 2019;11(4):217-218

23 Wong KW, Lai TC, Ng AT, et al. Anterior perineal hernia after anterior exenteration. Asian J Urol 2017;4(4):253-255

24 Balla A, Batista Rodríguez G, Buonomo N, et al. Perineal hernia repair after abdominoperineal excision or extralevator abdominoperineal excision: a systematic review of the literature. Tech Coloproctol 2017;21(5):329-336

25 Musters GD, Buskens CJ, Bemelman WA, Tanis PJ.Perineal wound healing after abdominoperineal resection for rectal cancer: a systematic review and meta-analysis. Dis Colon Rectum 2014 57(9):1129-1139

26 Sayers AE, Patel RK, Hunter IA. Perineal hernia formation following extralevator abdominoperineal excision. Colorectal Dis 2015;17(4):351-355

27 Sayers AE, Lee MJ, Smart N, Fearnhead NS; NASBO Steering Group. Optimizing collaborator recruitment and maintaining engagement via social media during large multicentre studies: lessons learned from the National Audit of Small Bowel Obstruction (NASBO). Colorectal Dis 2018;20(12):1142-1150

28 Bertrand $\mathrm{K}$, Lefevre JH, Creavin B, et al. The management of perineal hernia following abdomino-perineal excision for cancer. Hernia 2019

29 Pulikkottil BJ, Pezeshk RA, Daniali LN, Bailey SH, Mapula S, Hoxworth RE. Lateral abdominal wall defects: the importance of anatomy and technique for a successful repair. Plast Reconstr Surg Glob Open 2015;3(8):e481

30 Parikh KR, Al-Hawary M, Millet JD, Burney R, Finks J, Maturen K. Incisional hernia repair: what the radiologist needs to know. AJR Am J Roentgenol 2017;209(6): 1239-1246

31 Basta MN, Kozak GM, Broach RB, et al. Can we predict incisional hernia?: development of a surgery-specific decision-support interface. Ann Surg 2019;270(3):544-553

32 Lee L, Abou-Khalil M, Liberman S, Boutros M, Fried GM, Feldman LS. Incidence of incisional hernia in the specimen extraction site for laparoscopic colorectal surgery: systematic review and meta-analysis. Surg Endosc 2017;31(12): 5083-5093

33 Walming S, Angenete E, Block M, Bock D, Gessler B, Haglind E. Retrospective review of risk factors for surgical wound dehiscence and incisional hernia. BMC Surg 2017;17(1):19

34 Emby DJ, Aoun G. CT technique for suspected anterior abdominal wall hernia. AJR Am J Roentgenol 2003;181(2):431-433

35 Christy MR, Apostolides J, Rodriguez ED, Manson PN, Gens D, Scalea T. The component separation index: a standardized biometric identity in abdominal wall reconstruction. Eplasty 2012;12:e17 
36 Levi B, Zhang P, Lisiecki J, et al. Use of morphometric assessment of body composition to quantify risk of surgical-site infection in patients undergoing component separation ventral hernia repair. Plast Reconstr Surg 2014;133(4):559e-566e

37 Aguirre DA, Santosa AC, Casola G, Sirlin CB. Abdominal wall hernias: imaging features, complications, and diagnostic pitfalls at multi-detector row CT. Radiographics 2005;25(6):1501-1520

38 Cheung M-T, Chia N-H, Chiu W-Y. Surgical treatment of parastomal hernia complicating sigmoid colostomies. Dis Colon Rectum 2001;44(2):266-270

39 Rondelli F, Reboldi P, Rulli A, et al. Loop ileostomy versus loop colostomy for fecal diversion after colorectal or coloanal anastomosis: a meta-analysis. Int J Colorectal Dis 2009;24(5):479-488

40 El Mouhadi S, Tourabi C, Cazejust J, Azizi L, Menu Y, Arrivé L. CT and MRI features of ileostomies. AJR Am J Roentgenol 2011;196(3):577-584

41 Cingi A, Cakir T, Sever A, Aktan AO. Enterostomy site hernias: a clinical and computerized tomographic evaluation. Dis Colon Rectum 2006;49(10):1559-1563
42 Sjödahl R, Anderberg B, Bolin T. Parastomal hernia in relation to site of the abdominal stoma. Br J Surg 1988;75(4):339-341

43 Ortiz H, Sara MJ, Armendariz P, de Miguel M, Marti J, Chocarro C. Does the frequency of paracolostomy hernias depend on the position of the colostomy in the abdominal wall? Int J Colorectal Dis 1994;9(2):65-67

44 Holihan JL, Chen JS, Greenberg J, et al. Incidence of port-site hernias: a survey and literature review. Surg Laparosc Endosc Percutan Tech 2016;26(6):425-430

45 Owens M, Barry M, Janjua AZ, Winter DC. A systematic review of laparoscopic port site hernias in gastrointestinal surgery. Surgeon 2011;9(4):218-224

46 Clark LH, Soliman PT, Odetto D, et al. Incidence of trocar site herniation following robotic gynecologic surgery. Gynecol Oncol 2013;131(2):400-403

47 Hindman NM, Kang S, Parikh MS. Common postoperative findings unique to laparoscopic surgery. Radiographics 2014;34(1):119-138

48 Tonouchi H, Ohmori Y, Kobayashi M, Kusunoki M. Trocar site hernia. Arch Surg 2004;139(11):1248-1256 